\title{
CUBIC SURFACES WITH SPECIAL PERIODS
}

\author{
JAMES A. CARLSON AND DOMINGO TOLEDO
}

(Communicated by Lev Borisov)

\begin{abstract}
We study special values of the point in the unit ball (period) associated to a cubic surface. We show that this point has coordinates in $\mathbb{Q}(\sqrt{-3})$ if and only if the abelian variety associated to the surface is isogenous to the product of five Fermat elliptic curves. The proof uses an explicit formula for the embedding of the ball in the Siegel upper half plane. We give explicit constructions of abelian varieties with complex multiplication by fields of the form $K_{0}(\sqrt{-3})$, where $K_{0}$ is a totally real quintic field, which arise from smooth cubic surfaces. We include Sage code for finding such fields and conclude with a list of related problems.
\end{abstract}

\section{INTRODUCTION}

Consider a complex elliptic curve $E$ with equation

$$
y^{2}=4 x^{3}-g_{2} x-g_{3} .
$$

The endomorphism ring of such a curve always contains the integer dilations $z \mapsto$ $n z$. An elliptic curve with a larger endomorphism ring is said to have complex multiplication. The additional endomorphisms are of the form $z \mapsto \lambda z$, and it is easy to see that $\lambda$ lies in a purely imaginary quadratic extension $K=\mathbb{Q}(\sqrt{-d})$ of the rational numbers. This number field can be identified with $E n d(E) \otimes \mathbb{Q}$ and is called the $C M$ field of $E$. Conversely, if $K$ is a purely imaginary quadratic extension of the rational numbers, an elliptic curve with $\mathrm{CM}$ field $K$ can be constructed as $\mathbb{C} / \mathcal{O}_{K}$, where $\mathcal{O}_{K}$ is the ring of integers in $K$.

Now fix a symplectic homology basis $\left\{\gamma_{1}, \gamma_{2}\right\}$, and let

$$
\lambda_{i}=\int_{\gamma_{i}} \frac{d x}{y}
$$

be the fundamental periods. If we write (1.1) as

$$
y^{2}=4\left(x-e_{1}\right)\left(x-e_{2}\right)\left(x-e_{3}\right),
$$

then the fundamental periods are the elliptic integrals

$$
\lambda_{i}=\int_{e_{i}}^{e_{i+1}} \frac{d x}{\sqrt{\left(x-e_{1}\right)\left(x-e_{2}\right)\left(x-e_{3}\right)}}, \text { for } i=1,2 .
$$

A theorem of Siegel (1932) tells us that if the coefficients $g_{i}$ are algebraic numbers, then at least one of the periods is transcendental, and in 1934, Schneider showed that the nonzero periods are always transcendental. For a specific example, consider

Received by the editors April 10, 2011 and, in revised form, October 2, 2011.

2010 Mathematics Subject Classification. Primary 14D07, 14K22.

This research was partially supported by NSF Grant DMS-0600816. The first author also gratefully acknowledges the support of the Clay Mathematics Institute and of CIMAT. 
the Fermat elliptic curve, with affine equation $y^{2}=4\left(x^{3}-1\right)$. Then with $e_{1}, e_{2}, e_{3}=$ $1, \omega, \omega^{2}$, in that order,

$$
\lambda_{2}=\int_{1}^{\infty} \frac{d x}{\sqrt{x^{3}-1}}=\frac{1}{3} B(1 / 6,1 / 2)=\frac{\Gamma(1 / 3)^{3}}{2^{4 / 3} \pi} .
$$

See [11, equation (10)]. Here, and throughout this paper, $\omega=e^{2 \pi i / 3}$, a primitive cube root of unity.

When $E$ has complex multiplication, the period ratio

$$
\tau=\lambda_{2} / \lambda_{1}
$$

lies in the $\mathrm{CM}$ field. In this case, the transcendence degree of the field generated by the periods, $\mathbb{Q}\left(\lambda_{1}, \lambda_{2}\right)$, is one. It is conjectured that in the non-CM case, the field generated by the periods is of transcendence degree two.

Consider next a cubic surface $S$. Because the Hodge structure on $H^{2}(S)$ is entirely of type $(1,1)$, there are no periods of interest. However, there is an auxiliary Hodge structure of weight three associated to $S$ which does have interesting periods and which behaves in many ways like the Hodge structure of an elliptic curve. This is the Hodge structure of a three-sheeted cover $T$ of projective 3 -space branched along $S$. The period domain for such Hodge structures is $\mathbb{B}^{4}$, the unit ball in complex four-space, which is the same as the complex hyperbolic space $\mathbb{C} H^{4}$. In this paper we use the notation $\mathbb{B}^{4}$ rather than $\mathbb{C} H^{4}$.

There is a natural period map

$$
\mathcal{P}: \mathcal{M}_{s t} \longrightarrow \Gamma \backslash \mathbb{B}^{4}
$$

from the moduli space of stable cubic surfaces (those with at worst nodal singularities) to the indicated quotient of the ball by an arithmetic group. It is an isomorphism 3. The aim of this paper is to explore the relation between cubic surfaces and their periods. The theory of elliptic curves serves as a model of what might be possible to establish, at least in part. In particular, we will investigate the rationality of period ratios. While the results presented in this direction are modest, they point to several interesting questions. We discuss them at the end of this paper.

\section{Statement of Results}

We first review the main results of 3 and explain the mapping (1.2). We summarize the results we need; details can be found in [3] and in [5].

2.1. Standard notation. The following notation will be used throughout this paper. If $S \subset \mathbb{P}^{3}$ is a cubic surface defined by a cubic equation $F(x)=0$, let $T$ be the cubic threefold given by $\left\{y^{3}=F(x)\right\} \subset \mathbb{P}^{4}$, where $x \in \mathbb{C}^{4}$ and $y \in \mathbb{C}$. Let $\omega$ denote the primitive cube root of unity $\omega=\exp (2 \pi i / 3)$, and let $\sigma$ be the automorphism of $T$ over $\mathbb{P}^{3}$ defined by $\sigma(x, y)=(x, \omega y)$. Finally, let $\theta=i \sqrt{3}$ be the square root of -3 situated on the positive imaginary axis in the complex plane.

2.2. The Eisenstein structure of $T$. The middle cohomology $H^{3}(T, \mathbb{Z})$ is a free abelian group of rank 10 with an automorphism $\sigma$ satisfying $\sigma^{2}+\sigma+1=0$; hence it is a free module of rank 5 over the $\operatorname{ring} \mathcal{E}=\mathbb{Z}[\omega]$ of Eisenstein integers. This module carries a natural $\mathcal{E}$-valued Hermitian form $h$ of signature $(4,1)$ and determinant one given by

$$
-2 h(x, y)=\left\langle\left(\sigma-\sigma^{-1}\right) x, y\right\rangle+\left(\omega-\omega^{-1}\right)\langle x, y\rangle
$$


(see (2.3.1) of [3]) or, more simply, by the equivalent formula

$$
h(x, y)=\langle x, \sigma y\rangle-\omega\langle x, y\rangle
$$

as in (2.1) of [5]. This form is standard: $\left(H^{3}(T, \mathbb{Z}), \sigma, h\right)$ is isomorphic to $\left(\mathcal{E}^{5}, \omega, h_{0}\right)$, where $h_{0}(z, w)=-z_{0} \bar{w}_{0}+z_{1} \bar{w}_{1}+\cdots+z_{4} \bar{w}_{4}$.

2.3. The Hodge structure of $T$. Since $H^{3,0}(T)=0$, the Hodge structure has the form $H^{2,1} \oplus H^{1,2}$. The symmetry $\sigma$ is an automorphism of $H^{3}=H^{3}(T, \mathbb{C})$ with eigenvalues $\omega$ and $\bar{\omega}$. Let $H^{3}=H_{\omega}^{3} \oplus H_{\bar{\omega}}^{3}$ be the eigenspace decomposition. The summands are in natural duality by the cup-product pairing and are interchanged by complex conjugation. Because $\sigma$ is holomorphic, its action on cohomology is compatible with the Hodge decomposition. Thus one has complex Hodge structures

$$
H_{\omega}^{2,1} \oplus H_{\omega}^{1,2} \text { on } H_{\omega}^{3} \quad \text { and } \quad H_{\bar{\omega}}^{2,1} \oplus H_{\bar{\omega}}^{1,2} \text { on } H_{\bar{\omega}}^{3},
$$

where the summands have dimensions $4,1,1,4$ in the order listed. Equivalently, the same summands give the eigenspace decompositions

$$
H^{2,1}=H_{\omega}^{2,1} \oplus H_{\bar{\omega}}^{2,1} \text { and } H^{1,2}=H_{\omega}^{1,2} \oplus H_{\bar{\omega}}^{1,2} .
$$

The Hermitian form

$$
h^{\prime}(\alpha, \beta)=-i \sqrt{3}\langle\alpha, \bar{\beta}\rangle
$$

on $H^{3}(T, \mathbb{C})$ is positive definite on $H^{2,1}$, negative definite on $H^{1,2}$ and these two subspaces are $h^{\prime}$-orthogonal. There are complex linear isomorphisms

$$
\left(H_{\omega}^{3}, h^{\prime}\right) \cong\left(\left(H^{3}(T, \mathbb{Z}), \sigma\right) \otimes_{\mathcal{E}} \mathbb{C}, h\right) \cong \mathcal{E}^{4,1} \otimes_{\mathcal{E}} \mathbb{C} \cong \mathbb{C}^{4,1}
$$

and

$$
\left(H_{\bar{\omega}}^{3}, h^{\prime}\right) \cong\left(\left(H^{3}(T, \mathbb{Z}), \sigma^{-1}\right) \otimes_{\mathcal{E}} \mathbb{C},-\bar{h}\right) \cong \mathcal{E}^{1,4} \otimes_{\mathcal{E}} \mathbb{C} \cong \mathbb{C}^{1,4},
$$

where $\mathcal{E}^{4,1}$ is the standard model $\left(\mathcal{E}^{5}, \omega, h_{0}\right)$ above, and $\mathcal{E}^{1,4}$ is $\left(\mathcal{E}^{5}, \omega,-\bar{h}_{0}\right)$. Thus the decomposition $H^{3}(T)=H_{\omega}^{3} \oplus H_{\bar{\omega}}^{3}$ is a decomposition

$$
\mathbb{C}^{5,5}=\mathbb{C}^{4,1} \oplus \mathbb{C}^{1,4},
$$

and the integral submodule $\mathbb{Z}^{10} \subset \mathbb{C}^{10}$ corresponding to $H^{3}(T, \mathbb{Z}) \subset H^{3}(T, \mathbb{C})$ projects to Eisenstein lattices $\mathcal{E}^{4,1}, \mathcal{E}^{1,4}$ in each summand.

The Hodge structure on $H^{3}(T)$ is determined by either subspace $H_{\omega}^{2,1} \subset H_{\omega}^{3}$ or $H_{\bar{\omega}}^{2,1} \subset H_{\bar{\omega}}^{3}$, since each subspace determines the other by conjugation and orthogonality. Either collection constitutes a ball $\mathbb{B}^{4}$ in a Grassmannian, namely the positive hyperplanes in $\mathbb{C}^{4,1}$, respectively the positive lines in $\mathbb{C}^{1,4}$. We choose the second description for the $\mathbb{B}^{4}$ of (1.2) with the consequent description of $\Gamma=$ $P U(1,4, \mathcal{E})$, the projectivized group of $\mathcal{E}$-linear isometries of $\mathcal{E}^{1,4}$.

2.4. The period map. The period map $\mathcal{P}$ is defined on a covering space of the space of cubic forms. The points of this covering space correspond to a way of choosing the isomorphisms in (2.3), which is called a framing in (3.9) of [3]. If $\phi$ is the chosen isomorphism, $\mathcal{P}(F, \phi)=\phi\left(H_{\omega}^{2,1}\right) \subset \mathbb{C}^{1,4}$. It descends to the map $\mathcal{P}$ of (1.2).

Note that the choice of Hermitian form in (2.4) of [3] is the negative of the standard choice (2.1). Consequently, we have to make all the obvious sign changes when quoting [3]. Note also that the isomorphisms (2.2) and (2.3) are complex linear and incorporate the correction added in proof to $(2.2)$ of 3 . Our choice is equivalent to Beauville's choice of a positive hyperplane in $\mathbb{C}^{4,1}$, and $\mathcal{P}$ is holomorphic. 
2.5. The intermediate Jacobian. This period map determines $J(T)$, the intermediate Jacobian of $T$, which is a five-dimensional principally polarized abelian variety, thus classified by a point in the moduli space $\mathcal{A}_{5}=S p(10, \mathbb{Z}) \backslash \mathbb{H}_{5}$, where $\mathbb{H}_{5}$ is the Siegel upper half plane of genus 5 . Taking a standard model $\left(\mathbb{Z}^{10},\langle\rangle,\right)$ of a free abelian group of rank 10 with integral symplectic form $\langle$,$\rangle and defin-$ ing the Hermitan form $h^{\prime}$ on $\mathbb{Z}^{10} \otimes \mathbb{C}=\mathbb{C}^{10}=\mathbb{C}^{5,5}$ as above, $\mathbb{H}_{5}$ is given as the space of positive Lagrangian subspaces. These subspaces of $\mathbb{C}^{10}$ are 5-dimensional, $\langle$, $\rangle$-isotropic, and $h^{\prime}$-positive. We obtain a map $\iota: \mathbb{B}^{4} \longrightarrow \mathbb{H}_{5}$ defined by

$$
\iota(l)=\bar{l}^{\perp} \oplus l \subset \mathbb{C}^{5,5}=\mathbb{C}^{4,1} \oplus \mathbb{C}^{1,4} ;
$$

see (9.2.1) of [3] and (4.2) of [5]. This gives a holomorphic, totally geodesic embedding $\iota: \mathbb{B}^{4} \longrightarrow \mathbb{H}_{5}$, which is equivariant with respect to the natural homomorphism $\lambda: \Gamma \rightarrow S p(10, \mathbb{Z})$ given by $\lambda(\gamma)=\bar{\gamma} \oplus \gamma={ }^{t} \gamma^{-1} \oplus \gamma$ in the decomposition (2.4) into spaces that are simultaneously dual and conjugate to each other (transposition is with respect to the complex bilinear form $\left.h_{0}(z, \bar{w})\right)$. The image of $\lambda$ is the centralizer of $\sigma$ in $S p(10, \mathbb{Z})$. It follows that $\iota$ descends to an embedding $\iota: \Gamma \backslash \mathbb{B}^{4} \longrightarrow \mathcal{A}_{5}$, and the image of $\iota$ consists of those principally polarized abelian varieties on which $\sigma$ acts with signature $(4,1)$.

2.6. The hyperplane arrangement. The group $\Gamma$ is generated by complex reflections of order 6 (hexaflections) on the hyperplanes $v^{\perp}$, where $v \in \mathcal{E}^{1,4}$ and $h_{0}^{*}(v)=-1$, where $h_{0}^{*}=-\bar{h}_{0}$ is the standard form on $\mathbb{C}^{1,4}$; see $\S 7$ of 3 . Let $\mathcal{H} \subset \mathbb{B}^{4}$ be defined by

$$
\mathcal{H}=\bigcup\left\{\mathbb{B}^{3}\left(v^{\perp}\right): v \in \mathcal{E}^{1,4}, h_{0}^{*}(v)=-1\right\},
$$

where $\mathbb{B}^{3}\left(v^{\perp}\right) \subset \mathbb{B}^{4}$ is the ball of positive lines of the $(1,3)$-form $h_{0}^{*} \mid v^{\perp}$. This is a locally finite collection of hyperplanes and the group $\Gamma$ acts transitively on its elements. Moreover, $\mathcal{H}$ is an orthogonal arrangement of hyperplanes, meaning that any two elements are either disjoint or meet at right angles; see (7.29) of [3]. In particular, given any $x \in \mathbb{B}^{4}$, there can be at most 4 hyperplanes in this collection that contain $x$.

2.7. Explicit formula for period map. To get an explicit formula for the period map, let $\gamma=\left\{\gamma_{0}, \ldots, \gamma_{4}\right\}$ be a basis, as a $\mathbb{Z}[\omega]$-module, for the dual space $H_{3}(T, \mathbb{Z})$ of $H^{3}(T, \mathbb{Z})$, for which the dual Hermitian form is diagonal with diagonal entries $(-1,+1,+1,+1,+1)$. (This choice is equivalent to a framing as in (3.9) of [3].) Let $\Phi=\Phi(F)$ be the nonzero element $\Omega / F^{4 / 3}$ of $H_{\bar{\omega}}^{2,1}$ associated to the cubic form $F$ as in (3) of Theorem (6.5) of [3]. Then the period vector associated to these choices is the vector

$$
\mathcal{P}(F, \gamma)=\left(\int_{\gamma_{0}} \Phi, \ldots, \int_{\gamma_{4}} \Phi\right) .
$$

More explicitly, let $f(x, y, z)=0$ be an affine equation for the cubic surface in one of the standard affine open sets of $\mathbb{P}^{3}$. Then $w^{3}=f(x, y, z)$ is an affine equation for the cubic threefold, and the natural generator $\Phi(F)$ for $H_{\bar{\omega}}^{2,1}(T)$ is simply

$$
\Phi=\frac{d x \wedge d y \wedge d z}{w^{4}}
$$

This is the analogue of the abelian differential $d x / y$ for an elliptic curve. 


\subsection{The main theorem of 3 .}

○ The map $\mathcal{P}$ of (2.5) descends to a map $\mathcal{P}: \mathcal{M}_{s m} \longrightarrow \Gamma \backslash \mathbb{B}^{4}$ of the quotient spaces and extends to the map $\mathcal{P}: \mathcal{M}_{s t} \longrightarrow \Gamma \backslash \mathbb{B}^{4}$ of (1.2), which is an isomorphism.

- Moreover, if $\mathcal{M}_{s m}$ is the moduli space of smooth cubic surfaces, then its restriction to $\mathcal{M}_{s m}$ gives an isomorphism

$$
\mathcal{P}: \mathcal{M}_{s m} \rightarrow \Gamma \backslash\left(\mathbb{B}^{4}-\mathcal{H}\right) .
$$

- Finally, if $\mathcal{M}_{\text {nod }}$ is the moduli space of nodal cubic surfaces, then $\mathcal{M}_{s t}=$ $\mathcal{M}_{s m} \cup \mathcal{M}_{\text {nod }}$ and the restriction of the period map to $\mathcal{M}_{\text {nod }}$ gives an isomorphism

$$
\mathcal{P}: \mathcal{M}_{\text {nod }} \rightarrow \Gamma \backslash \mathcal{H}=\Gamma_{v} \backslash \mathbb{B}^{3}\left(v^{\perp}\right)=\Gamma_{0} \backslash \mathbb{B}^{3},
$$

where $v$ is any fixed element of $\mathcal{E}^{1,4}$ with $h_{0}^{*}(v)=-1$. For example, if $v=(0,1,0,0,0)$ and $\Gamma_{v}$ is its stabilizer in $\Gamma$, then $\Gamma_{0}$ is the group $P U(1,3, \mathcal{E})$ acting on $\mathbb{B}^{3}$.

2.9. The abelian varieties. We need one final result that is not explicitly stated in 3 but follows easily from that paper. In fact, it is the underlying philosophy of proofs of the results just quoted.

- A principally polarized abelian variety $A \in \mathcal{A}_{5}$ is in the image $\iota\left(\Gamma \backslash \mathbb{B}^{4}\right)=$ $\iota \mathcal{P}\left(\mathcal{M}_{s t}\right)$ if and only if it has an automorphism $\sigma$ of order 3 of type $(4,1)$, meaning that the induced action on $H^{1,0}(A)$ has eigenvalue $\omega$ of multiplicity 4 and $\bar{\omega}$ of multiplicity 1 .

- Furthermore, if $A \in \iota \mathcal{P}\left(\mathcal{M}_{s t}\right)$ as above, then $A \in \iota \mathcal{P}\left(\mathcal{M}_{s m}\right)$ if and only if $A$ is, in addition, irreducible.

- Similarly, if $A \in \iota \mathcal{P}\left(\mathcal{M}_{s t}\right)$ as above, then $A \in \iota \mathcal{P}\left(\mathcal{M}_{\text {nod }}\right)$ if and only if $A$ is reducible and contains at least one irreducible factor isomorphic to a Fermat elliptic curve.

The proof of the first statement follows from the last statement of (2.5) and the fact that all automorphisms of type $(4,1)$ are conjugate under $S p(10, \mathbb{Z})$. The second and third statements follow from the fact that the intermediate Jacobian of a nonsingular cubic threefold is irreducible, while each nodal surface gives rise to an $A_{2}$-singularity in the associated threefold, which is turn gives a summand of the Hodge structure of a Fermat elliptic curve. See Lemmas (5.4), (5.7) of [3] for the last point.

2.10. New results. Given this background, we now come to the results of this paper. If $f$ has coefficients in a field $K$, then so does $\Phi$ : it is a $K$-rational differential. Consequently $\Phi$ is well-defined up to a nonzero element of $K$. Therefore questions about the rationality of periods, referring to such a $K$-rational differential, make sense for any field $L$ extending $K$.

There is a philosophy that periods of $K$-rational differentials are almost always transcendental; this is, however, difficult to prove in any concrete instance. Thus our focus will be on period ratios such as $\omega_{2} / \omega_{1}$ for cubic curves or

$$
v_{i}=\frac{\int_{\gamma_{i}} \Phi}{\int_{\gamma_{0}} \Phi}
$$


for cubic surfaces. In the last ratio, we may rescale $\Phi$ so that $v_{0}=1$. The resulting periods $v_{i}$, which are now relative to a differential with unknown rationality properties, should be thought of as period ratios. This will often be our point of view in what follows.

Let us write the period vector as $v=\left(a, b_{1}, b_{2}, b_{3}, b_{4}\right)=(a, b)$. When $a=1$, we say that the period vector is normalized. In this case, since $(a, b)$ is a positive vector for $h_{0}^{*}$, we have $|a|^{2}-|b|^{2}>0$, so $b$ is a vector in $\mathbb{C}^{4}$ of length at most 1 . The corresponding Hodge structure of level one and genus five is determined by a $5 \times 10$ matrix of periods $P=(A, B)$, where $P$ represents $\iota(a, b)$. In the usual terminology, $P$ is normalized in the case that $A$ is the identity (in which case $B \in \mathbb{H}_{5}$; that is, $B$ is symmetric with positive-definite imaginary part). Subsection 2.9 tells us that, in the presence of the action by $\sigma$, the period vector and the period matrix determine each other, up to natural equivalences (action of $\Gamma$ and $S p(10, \mathbb{Z})$ ).

Finally, recall that an abelian variety over $\mathbb{C}$ is said to be of CM-type if (a) it is isogeneous to a product $A_{1} \times \cdots \times A_{r}$ of simple abelian varieties and (b) there are fields $K_{i} \subset \operatorname{End}\left(A_{i}\right) \otimes \mathbb{Q}$ such that $\left[K_{i}: \mathbb{Q}\right] \geq 2 \operatorname{dim} A_{i}$ (in which case $\left[K_{i}: \mathbb{Q}\right]=2 \operatorname{dim} A_{i}$ and $\left.K_{i}=\operatorname{End}\left(A_{i}\right) \otimes \mathbb{Q}\right)$. If the fields $K_{i}$ are equal, we say that $K=K_{i}$ is the CM field of the abelian variety. See Mumford [9, p. 347]. We can now state our first result.

Theorem 2.1. Let $S$ be a cubic surface and let $J$ be its abelian variety. The following are equivalent:

(a) one (and hence all) normalized period vectors of $S$ have coefficients in $\mathbb{Q}(\omega)$;

(b) one (and hence all) normalized period matrices of $S$ have coefficients in $\mathbb{Q}(\omega)$;

(c) $J$ is isogeneous to a product of Fermat elliptic curves.

It follows from the theorem that for a cubic surface with period vector in $\mathbb{Q}(\omega)$, $\operatorname{End}(J) \otimes_{\mathbb{Z}} \mathbb{Q}$ is the ring of $5 \times 5$ matrices with coefficients in $\mathbb{Q}(\omega)$; as a consequence of the main theorem, we see that cubic surfaces with period vector in $\mathbb{Q}(\omega)$ are of CM-type with CM field $\mathbb{Q}(\omega)$. In particular, we get the following well-known fact:

Corollary 2.2. The Hodge structures of CM type in $\mathbb{B}^{4}$ are dense.

Because the period map (1.2) is surjective, there is a dense set of smooth cubic surfaces with periods in $\mathbb{Q}(\omega)$. As noted in [3, Theorem 11.6, 11.9], some explicit surfaces with periods of this kind are known. A period vector (not normalized) of the Fermat cubic surface $x^{3}+y^{3}+z^{3}+w^{3}=0$ is

$$
v=(2-\bar{\omega}, 1,1,1,1),
$$

and that of the diagonal cubic surface $x^{3}+y^{3}+z^{3}+w^{3}+u^{3}=0, x+y+x+w+u=0$ is

$$
v=(3,1,1,1,1) .
$$

Again, it is more convenient to write this vector in nonnormalized form. The rationality properties of the differential form which gives the periods are unknown. Consequently the periods should be thought of as period ratios.

It is natural to ask how to characterize cubic surfaces with periods in $\mathbb{Q}(\omega)$ in purely geometric terms. We do not know the answer, even conjecturally. 
In a different direction, we state our last result, which concerns the opposite extreme of complex multiplication, namely cubic surfaces whose CM algebra is a field:

Theorem 2.3. There exist simple, principally polarized abelian varieties $A$ of $d i$ mension five with three-fold symmetry $\sigma$ of type $(4,1)$ and with rational endomorphism ring $\operatorname{End}(A) \otimes \mathbb{Q}$ isomorphic to a $C M$-field $K$ of the form $K_{0}(\sqrt{-3})$, where $K_{0}$ is a totally real field of degree five. The Hodge structure of $A$ is rational over $K$, and it is defined by a period vector rational over the same field. By subsection 2.9, $A$ is the abelian variety of a smooth cubic surface.

Remark. We study extreme cases of CM types. In [2, Section 3], Achter classifies, up to isogeny, all possible CM types, endomorphism algebras, and Mumford-Tate groups of abelian five-folds which admit an action of $\mathbb{Z}[\omega]$.

\section{Cubic surfaces with period vector rational over $\mathbb{Q}(\omega)$}

The key to the proof of the first theorem is an analysis of the periods of the most singular stable cubic surface. We begin with the following:

Proposition 3.1. Any normalized period vector of the Cayley cubic surface

$$
\frac{1}{x}+\frac{1}{y}+\frac{1}{z}+\frac{1}{w}=0
$$

is $\Gamma$-equivalent to $v=(1,0,0,0,0)$.

Proof. The Cayley cubic surface is a cubic surface with 4 nodes. It is classically known to be the surface with the maximum possible number of nodes. Thus, by the last part of subsection 2.8, it lies in the intersection of 4 distinct hyperplanes in $\mathcal{H}$. One such point is $(1,0,0,0,0)$, which is the intersection of $(0,1,0,0,0)^{\perp}, \ldots$, $(0,0,0,0,1)^{\perp}$. It is easy to see that these are precisely the points in $v \in \mathcal{E}^{1,4}$ with $h_{0}^{*}(v)=1$ and that all such points are equivalent under $\Gamma$. Thus the period of the Cayley cubic is the $\Gamma$-orbit of $(1,0,0,0,0)$.

Note that this argument implies the well-known classical fact that all 4-nodal cubic surfaces are isomorphic over the complex numbers. Also subsection 2.8 combined with subsection 2.6 reproves the classical fact quoted in this proof that 4 is the maximum number of nodes.

As we show in a moment, the formula we will derive for the period matrix in terms of the period vector will give:

Corollary 3.2. Any normalized period matrix of the Cayley cubic surface is $\Gamma$ equivalent to

$$
P=(I, \omega I),
$$

where $I$ is the identity matrix. Thus the intermediate Jacobian of the Cayley cubic surface is the product of five Fermat elliptic curves.

As a general principle, the period vector in our context determines the period matrix and conversely.

Let us now compute the period matrix $P$ associated to a general cubic surface $S$ with equation $F=0$, that is, the period matrix of the intermediate Jacobian of the associated cyclic cubic threefold $T$ as in subsection 2.1. To this end, recall from subsection 2.7 that $\gamma=\left\{\gamma_{0}, \ldots, \gamma_{4}\right\}$ is a unitary basis of $H_{3}(T, \mathbb{Z})$ as a Hermitian 
Eisenstein module. Let $\gamma^{\prime}=\left\{\gamma_{0}^{\prime}, \ldots, \gamma_{4}^{\prime}\right\}=\left\{\sigma^{-1} \gamma_{0}, \sigma \gamma_{1}, \ldots, \sigma \gamma_{4}\right\}$. The homology basis $\gamma \cup \gamma^{\prime}$ is a sympletic basis with respect to the standard form

$$
J=\left(\begin{array}{rr}
0 & I \\
-I & 0
\end{array}\right)
$$

where $I$ is the identity matrix. Choose a basis $\left\{\Phi^{0}, \ldots, \Phi^{4}\right\}$ for $H^{2,1}$ where $\Phi^{0} \in$ $H_{\bar{\omega}}^{2,1}$ and $\Phi^{i} \in H_{\omega}^{2,1}$ for $i>0$. Then the period matrix takes the form

$$
P=(A, B),
$$

where

$$
A_{i j}=\int_{\gamma_{j}} \Phi^{i}, \quad B_{i j}=\int_{\gamma_{j}^{\prime}} \Phi^{i} .
$$

The change of variable formula in the calculus coupled with the fact that the $\Phi^{i}$ are eigenvectors of $\sigma$ implies that

$$
\int_{\gamma_{j}^{\prime}} \Phi^{i}=\lambda \int_{\gamma_{j}} \Phi^{i}
$$

where $\lambda \in\{\omega, \bar{\omega}\}$. We may choose the basis $\left\{\Phi^{1}, \ldots, \Phi^{4}\right\}$ so that $A_{i j}=\delta_{i j}$ for $i, j \in\{1, \ldots, 4\}$. The first Riemann bilinear relation determines the first column of $A$ in terms of the second, while (3.3) determines $B$ in terms of $A$. We conclude that

$$
P=\left(\begin{array}{cccccccccc}
1 & b_{1} & b_{2} & b_{3} & b_{4} & \omega & \bar{\omega} b_{1} & \bar{\omega} b_{2} & \bar{\omega} b_{3} & \bar{\omega} b_{4} \\
b_{1} & 1 & 0 & 0 & 0 & \bar{\omega} b_{1} & \omega & 0 & 0 & 0 \\
b_{2} & 0 & 1 & 0 & 0 & \bar{\omega} b_{2} & 0 & \omega & 0 & 0 \\
b_{3} & 0 & 0 & 1 & 0 & \bar{\omega} b_{3} & 0 & 0 & \omega & 0 \\
b_{4} & 0 & 0 & 0 & 1 & \bar{\omega} b_{4} & 0 & 0 & 0 & \omega
\end{array}\right) .
$$

In the case of the Cayley cubic surface, Proposition 3.1 gives that the parameter vector $b$ is zero and the period matrix is

$$
P=(I, \omega I),
$$

where $I$ is the identity matrix. It follows that the abelian variety of the Cayley cubic surface is the product of five Fermat elliptic curves. This proves Corollary 3.2.

The map $\psi(b)=Z$, where $Z=B A^{-1}$ and $A, B$ are as in (3.2), gives a matrix formula for the imbedding $\iota$ of (2.5) of the unit ball in the Siegel upper half space of genus five. It can be written more concisely as

$$
P(b)=\left(\begin{array}{cccc}
1 & b & \omega & \bar{\omega} b \\
{ }^{t} b & I & \bar{\omega}^{t} b & \omega I
\end{array}\right) .
$$

To understand better the location of $Z(b)$ in the Siegel upper half space, note that the quantity $\delta=\operatorname{det} A=1-b \cdot b$ is nonzero since $|b|^{2}<1$. Thus, if $P=(A, B)$ is the period matrix, we can form $A^{-1}(A, B)=(1, Z)$, where $Z$ is the normalized matrix of $B$-periods, a symmetric matrix with positive definite imaginary part. The inverse of $A$ is given by the following expression:

$$
A^{-1}=\delta^{-1}\left[\begin{array}{ccccc}
1 & -b_{1} & -b_{2} & -b_{3} & -b_{4} \\
-b_{1} & \delta_{1} & b_{1} b_{2} & b_{1} b_{3} & b_{1} b_{4} \\
-b_{2} & b_{1} b_{2} & \delta_{2} & b_{2} b_{3} & b_{2} b_{4} \\
-b_{3} & b_{1} b_{3} & b_{2} b_{3} & \delta_{3} & b_{3} b_{4} \\
-b_{4} & b_{1} b_{4} & b_{2} b_{4} & b_{3} b_{4} & \delta_{4}
\end{array}\right]
$$


where $\delta=1-\left(b_{1}^{2}+b_{2}^{2}+b_{3}^{2}+b_{4}^{2}\right)$ is the determinant of $A$ and where $\delta_{i}=\delta+b_{i}^{2}$. Then

$$
Z=A^{-1} B=\delta^{-1}\left[\begin{array}{ccccc}
\delta_{0}^{\prime} & -\theta b_{1} & -\theta b_{2} & -\theta b_{3} & -\theta b_{4} \\
-\theta b_{1} & \delta_{1}^{\prime} & \theta b_{1} b_{2} & \theta b_{1} b_{3} & \theta b_{1} b_{4} \\
-\theta b_{2} & \theta b_{1} b_{2} & \delta_{2}^{\prime} & \theta b_{2} b_{3} & \theta b_{2} b_{4} \\
-\theta b_{3} & \theta b_{1} b_{3} & \theta b_{2} b_{3} & \delta_{3}^{\prime} & \theta b_{3} b_{4} \\
-\theta b_{4} & \theta b_{1} b_{4} & \theta b_{2} b_{4} & \theta b_{3} b_{4} & \delta_{4}^{\prime}
\end{array}\right]
$$

where

$$
\delta_{0}^{\prime}=\omega-\bar{\omega}\left(b_{1}^{2}+b_{2}^{2}+b_{3}^{2}+b_{4}^{2}\right)
$$

and

$$
\delta_{i}^{\prime}=\omega \delta-\theta b_{i}^{2} .
$$

Rewriting the matrix $Z$, we obtain the following proposition:

Proposition 3.3. Let $b \in \mathbb{B}^{4}$ be a row vector (thus $|b|<1$ ), let ${ }^{t} b$ be the corresponding column vector, and let $b \otimes b$ be the matrix whose $i j$-th entry is $b_{i} b_{j}$. Then

$$
Z(b)=\omega I+(\theta / \delta)\left(\begin{array}{cc}
b \cdot b & -b \\
-{ }^{t} b & b \otimes b
\end{array}\right)
$$

is a function of $b$ that maps points of the unit ball to points of the Siegel upper half space, with the origin of the ball mapped to the normalized period matrix of the product of five Fermat elliptic curves. It gives a matrix formula for the imbedding $\iota: \mathbb{B}^{4} \longrightarrow \mathbb{H}_{5}$ defined in subsection 2.5 .

Proof of Theorem 2.1. The proof proceeds as follows. (1) The isogeny class of an abelian variety with lattice $\Lambda \subset \mathbb{C}^{n}$ is the isomorphism class of the embedding $\Lambda \otimes \mathbb{Q} \subset \mathbb{C}^{n}$ (isomorphism by complex linear maps of $\mathbb{C}^{n}$ ). (2) If $\Lambda_{0}$ is the lattice of $E^{5}$, where $E$ is the Fermat elliptic curve, then $\Lambda_{0} \otimes \mathbb{Q}=\mathbb{Q}(\omega)^{5} \subset \mathbb{C}^{5}$. (3) If the period vector $b$ is in $\mathbb{Q}(\omega)$, then formula (3.4) for the period matrix shows that the columns of the matrix, which give a basis for the lattice $\Lambda$, have entries in $\mathbb{Q}(\omega)$. Thus $\Lambda \otimes \mathbb{Q}$ is isomorphic to $\Lambda_{0} \otimes Q$ as rational subspaces of $\mathbb{C}^{5}$.

\section{Proof of Theorem 2.3}

As we have just seen, one can distinguish certain points in the ball quotient, e.g., those whose period vector is rational over $\mathbb{Q}(\omega)$. In that case the abelian variety is isogenous to a product of Fermat elliptic curves.

To prove Theorem 2.3, we now seek special points in the ball quotient where the corresponding abelian variety is simple and where the rational endomorphism ring is a number field. To this end, consider first a totally real number field $K_{0}$ of degree $n$ and a purely imaginary quadratic extension, which we may write as $K=K_{0}(\sqrt{\delta})$ for some element $\delta$ in $K_{0}$. The field $K$ is a $C M$ field. It has $2 n$ distinct embeddings $\tau_{i}$ in the complex numbers. A $C M$ type for $K$ is a choice $\Phi=\left(\tau_{1}, \ldots, \tau_{n}\right)$, where $\tau_{i} \neq \bar{\tau}_{j}$ for any $i, j$. Let $\mathcal{O}_{K}$ be the ring of integers in $K$. Then one can form the complex torus $A(K, \Phi)=\mathbb{C}^{n} / \Phi\left(\mathcal{O}_{K}\right)$. Note that the ring $\mathcal{O}_{K}$ acts by endomorphisms on $A(K, \Phi)$, so that

$$
K \subset \operatorname{End}(A(K, \Phi)) \otimes \mathbb{Q} .
$$


If $A(K, \Phi)$ is simple, then the rational endomorphism ring is a division algebra of dimension at most $2 n$ over $\mathbb{Q}$. But $\operatorname{dim}_{\mathbb{Q}} K=2 n$, so

$$
K=\operatorname{End}(A(K, \Phi)) \otimes \mathbb{Q} .
$$

Thus the rational endomorphism ring of such a torus is the field $K$.

To polarize the torus $A(K, \Phi)$, we follow an argument of Mumford [8, page 212]. There he claims:

(*) the existence of an element $\alpha$ of $K$ such that $\tau_{i}(\alpha)=\sqrt{-1} \beta_{i}$, where the $\beta_{i}$ are positive reals.

Given such an element, the expression

$$
H(x, y)=2 \sum_{i=1}^{g} \beta_{i} \tau_{i}(x) \overline{\tau_{i}(y)}
$$

defines a positive Hermitian form on $K$. Its associated skew form is

$$
\Omega(x, y)=\Im H(x, y)=-2 \Re \sum_{i=1}^{g} \tau_{i}(\alpha) \tau_{i}(x) \overline{\tau_{i}(y)}=-\operatorname{Tr}_{K / \mathbb{Q}}(\alpha x \bar{y}) .
$$

Since $\alpha$ is an element of the field $K$, the trace form takes rational values. To ensure that the values of the form are integers, we choose $\alpha$ to be in $\mathcal{O}_{K}^{\vee}$, the lattice dual to $\mathcal{O}_{K}$.

To show the existence of the element $\alpha$ in $(*)$, Mumford argues as follows. Let $\delta$ be an element of $K_{0}$ such that $K=K_{0}(\sqrt{\delta})$. Since $K$ is a totally imaginary extension of the totally real field $K_{0}$, for each $i$ we can write $\tau_{i}(\sqrt{\delta})=\sqrt{-1} \gamma_{i}$, where $\gamma_{i}$ is a nonzero real number. One can also find an element $\eta \in K_{0}$ such that $\tau_{i}(\eta)$ and $\gamma_{i}$ have the same sign for all $i$. Then $\alpha=\eta \sqrt{\delta}$ is such that $\tau_{i}(\alpha)=\sqrt{-1} \beta_{i}$, where $\beta_{i}$ is a positive real number for all $i$. To summarize, we have the following:

Proposition 4.1. Given an element $\alpha \in \mathcal{O}_{K}^{\vee}$ satisfying $(*)$, the expression (4.2) defines a polarization of $A(K, \Phi)$.

There remains the question of whether this polarization, which is determined by a suitable element $\alpha \in K$, can be chosen to be principal. If $\alpha \in \mathcal{O}_{K}^{\vee}$ and in addition,

$$
\alpha \mathcal{O}_{K}=\mathcal{O}_{K}^{\vee}
$$

then the form $\Omega(x, y)$ is unimodular. (See [10, p. 195], where the dual as a module is identified with the dual with respect to the trace pairing.) The polarization (4.3) is principal exactly when condition (4.4) holds; this in turn holds if and only if $\alpha^{-1}$ generates the different of $K$ - the fractional ideal $\left(\mathcal{O}_{K}^{\vee}\right)^{-1}$. (Thus in the cases we consider, the different is a principal ideal.) To conclude, we have

Proposition 4.2. The polarization defined by $\alpha \in \mathcal{O}_{K}^{\vee}$ is principal if and only if $\alpha^{-1}$ generates the different of $K$.

In the case that $K=K_{0}(\sqrt{-3})$, one can restate the criterion $(*)$ in terms of the different of $K_{0}$. To this end we introduce the following notion.

Definition 4.3. Let $\beta$ be an element of a totally real field $K_{0}$ of degree $n$ over $\mathbb{Q}$. Let $\tau_{i}, i=1, \ldots, n$ be the imbeddings of $K_{0}$ in the real numbers. Let $\epsilon$ be an $n$-vector with entries \pm 1 . Then $\beta$ is $\epsilon$-positive if

$$
\epsilon_{i} \tau_{i}(\beta)>0 \text { for all } i \text {. }
$$


In the case that $\epsilon_{i}=+1$ for all $i, \epsilon$-positivity is the same as total positivity.

Proposition 4.4. Fix a vector $\epsilon=\left(\epsilon_{1}, \ldots, \epsilon_{n}\right)$ with $\epsilon_{i}= \pm 1$. Let $\Phi$ be a CM-type for $K$ which extends a set of embeddings $\tau_{i}: K_{0} \longrightarrow \mathbb{R}$ to embeddings $\tau_{i}: K \longrightarrow \mathbb{C}$ such that $\tau_{i}(\sqrt{-3})=\epsilon_{i} \theta$. Suppose that there is an $\epsilon$-positive element $\beta$ of $K_{0}$ whose inverse generates the different of $K_{0}$. Then $\alpha=-\beta \theta^{-1}$ defines a principal polarization of $A(K, \Phi)$.

Proof. The element $\alpha=-\beta \theta^{-1}$ is the product of inverses of generators of the different for $K_{0}$ and $\mathbb{Q}(\sqrt{-3})$. By the multiplicativity of the different, this product is an inverse of a generator of the different of $K$, that is, a generator of $\mathcal{O}_{K}^{\vee}$. See [10, Prop. 2.2, p. 195]. Now

$$
\tau_{i}(\alpha)=-\tau_{i}(\beta) \tau_{i}\left(\theta^{-1}\right)=\sqrt{-1}\left(\epsilon_{i} \tau_{i}(\beta) / \sqrt{3}\right) .
$$

The quantity in parentheses is positive if $\epsilon_{i} \tau_{i}(\beta)$ is positive, Thus both (4.4) and (*) are satisfied.

There remains the question of whether the conditions of $\epsilon$-positivity for a generator of the different of $K_{0}$ can ever be satisfied. The next result sets forth a criterion for its satisfaction which can sometimes be verified by computation. We will carry out such a computation in the next section.

Proposition 4.5. Let $\Phi$ and $\beta$ be as in the preceding proposition. Suppose that there is a unit $\eta$ of $\mathcal{O}_{K_{0}}$ such that $\tau_{i}(\eta)$ has the same sign as $\epsilon_{i} \tau_{i}(\beta)$. Then the element $\beta \eta$ is $\epsilon$-positive.

Proof. If the vectors $\left(\tau_{1}(\eta), \ldots, \tau_{n}(\eta)\right)$ and $\left(\epsilon_{i} \tau_{1}(\beta), \ldots, \epsilon_{n} \tau_{n}(\beta)\right)$ lie in the same octant, then the conditions of the previous proposition are satisfied.

Let us return to the case of $K=K_{0}(\sqrt{-3})$, where $K_{0}$ is a totally real quintic field, and consider the problem of whether there are choices so that $A(K, \Phi, \alpha)$ is a principally polarized abelian variety which has an automorphism of order three and such that the eigenvalues of that automorphism on the space of abelian differentials are $\omega$ with multiplicity four and $\bar{\omega}$ with multiplicity one.

To this end, let $\tau_{1}, \ldots, \tau_{5}$ be the imbeddings of $K_{0}$ in $\mathbb{R}$. Extend them to imbeddings of $K$ in $\mathbb{C}$ by requiring

$$
\tau_{1}(\sqrt{-3})=-\theta \text { and } \tau_{i}(\sqrt{-3})=\theta \text { for } i>1 .
$$

Then $\left(\tau_{1}, \ldots, \tau_{5}\right)$ is a CM type for $K$ with the property that $\Phi\left(\frac{-1+\sqrt{-3}}{2}\right)$ acts on $\mathbb{C}^{5}$ with eigenvalues $(\bar{\omega}, \omega, \omega, \omega, \omega)$. It follows that

$$
\operatorname{dim} H_{\bar{\omega}}^{1,0}(A(K, \Phi))=1 .
$$

Now we construct a field with the required properties. Let $\zeta=\exp (2 \pi \sqrt{-1} / 11)$ be a primitive 11-th root of unity. The totally real subfield of $\mathbb{Q}(\zeta)$ is $K_{0}=\mathbb{Q}(\rho)$, where

$$
\rho=\zeta+\zeta^{-1}=2 \cos (2 \pi / 11)=1.6825 \ldots
$$

It is the totally really quintic field of smallest discriminant, namely $11^{4}=14641$. It is not hard to see that $\mathbb{Q}(\zeta)$ is a CM field and that one can construct a simple abelian variety from it. See [6, p. 24]. What we need, however, is a simple abelian variety with a suitable action of $\omega$. To this end, we establish the following: 
Proposition 4.6. Let $K=K_{0}(\sqrt{-3})$. Let $\Phi$ be the $C M$ type extended from a vector of embedding $\left(\tau_{1}, \ldots, \tau_{5}\right)$ of $K_{0}$ as in equation (4.5). Then $A(K, \Phi)$ is a simple, principally polarized abelian variety. It therefore corresponds to a smooth cubic surface.

Proof. The class number of $K_{0}$ is one, so that all ideals in $\mathcal{O}_{K_{0}}$ are principal. The different of $\mathcal{O}_{K_{0}}$ is the ideal generated by

$$
\delta_{0}=-4 r^{4}+r^{3}+14 r^{2}+4 r-9,
$$

where $r=-\rho$ generates $K_{0}$. This element is not totally positive. The group of units of $\mathcal{O}_{K_{0}}$ is isomorphic to $C_{2} \times \mathbb{Z}^{4}$. Generators for the free abelian part of this group are

$$
u_{1}, u_{2}, u_{3}, u_{4}=r^{4}-3 r^{2}+1, r^{2}-r-1, r-1, r,
$$

so that an arbitrary unit has the form $\pm u_{1}^{j} u_{2}^{k} u_{3}^{\ell} u_{4}^{m}$. One finds that the element

$$
\delta=u_{4} \delta_{0},
$$

which also generates the different ideal, is $\epsilon$-positive for $\epsilon=(+,+,-,+,+)$. The element $\beta=\delta^{-1}$ is also $\epsilon$-positive, and it is the element we use to define the principal polarization. The embeddings of $\delta$ in $\mathbb{R}$ are

$$
\begin{gathered}
21.7307463515808 \\
4.26952134163076 \\
-1.91569396353523 \\
14.0542888631537 \\
5.86113740717001
\end{gathered}
$$

The corresponding embeddings of the generator $r$ of $K$ over $\mathbb{Q}$ are

$$
\begin{gathered}
-1.68250706566236 \\
-0.830830026003773 \\
0.284629676546570 \\
1.30972146789057 \\
1.91898594722899
\end{gathered}
$$

The criterion for $\epsilon$-positivity is satisfied, and the abelian variety $A(K, \Phi, \alpha)$ is principally polarized.

To conclude that $A(K, \Phi, \alpha)$ corresponds to a smooth cubic surface, following subsection [2.9, it only remains to show that it is irreducible. It will be enough to show that it is simple. To that end, consider the Galois group $G$ of $K / \mathbb{Q}$. It is a group of order ten. The subgroup $\operatorname{Gal}\left(K_{0} / \mathbb{Q}\right)$ is normal. Since it is of order five, it is cyclic. The subgroup $\operatorname{Gal}(\mathbb{Q}(\omega) / \mathbb{Q})$ is also normal, and since it is of order two, it is cyclic. A group of order ten with these structural features must be cyclic.

The subfields of $K$ are the fixed sets of subgroups of the Galois group. Since a cyclic group of order ten has only two nontrivial subgroups, the field $K$ has only two nontrivial subfields, namely, $K_{0}$ and $\mathbb{Q}(\omega)$. Once we know the subfields of $K$, Mumford's criterion for simplicity of $A(K, \Phi, \alpha)[8, \mathrm{pp} .213-14]$ is easy to apply. It suffices to show that there are $\tau_{i}$ and $\tau_{j}$ such that $\tau_{i}\left|\mathbb{Q}(\omega) \neq \tau_{j}\right| \mathbb{Q}(\omega)$. The condition on the $\tau$ 's holds by construction. See equation (4.5). 
We have a construction

$\{$ Certain totally real quintic number fields $\} \longrightarrow\left\{B^{4} / \Gamma\right\}$.

The period matrices that arise in this way are rational over $K$. Indeed, consider the formula (3.5) for the normalized period matrix $Z=\left(Z_{i j}\right)$, where $i$ and $j$ run from 0 to 4 . Then the period vector is

$$
b=(1 / \theta)\left(Z_{01}, Z_{02}, Z_{03}, Z_{04}\right) .
$$

If $Z_{i j}$ is rational over $K$, then so is $b$. The converse also comes from formula (3.5). The period vector could be rational over $K_{0}$ and still correspond to a period matrix rational over $K$; it cannot, however, be rational over $\mathbb{Q}$ or $\mathbb{Q}(\omega)$.

\section{Computations And EXPeriments}

Above we described a method to show that the totally real quintic field of discriminant 14641 defines a principally polarized abelian variety with three-fold symmetry of the correct type. The same method can be used to produce lists of quintic fields with this property. Using the Sage code below, for example, we show that (1) there are 414 totally real quintic fields of discriminant less than $10^{6} ;(2)$ none of these have class number greater than 1; (3) there are 412 fields which satisfy the hypotheses of Proposition 4.4: (4) the density of such fields in the indicated range is about 0.995 . Below is the data for the fields of discriminant $<10^{5}$. The second column is the discriminant of the field. The third column answers the question: Is there a cubic surface with the given discriminant?

\begin{tabular}{|c|c|c|}
\hline \multicolumn{2}{|c|}{ discr } & result \\
\hline 1 & 14641 & True \\
\hline 2 & 24217 & True \\
\hline 3 & 36497 & True \\
\hline 4 & 38569 & True \\
\hline 5 & 65657 & True \\
\hline 5 & 70601 & True \\
\hline & 81509 & True \\
\hline 8 & 81589 & True \\
\hline 9 & 89417 & True \\
\hline
\end{tabular}

Sage code. We first define a function to return the class number of the field $K_{0}=\mathbb{Q}[X] /(F[X])$ for a polynomial $F$ :

def classNumber(F):

R. $\langle\mathrm{x}>=$ PolynomialRing (QQ)

$\mathrm{f}=\mathrm{R}(\mathrm{F})$

K. $\langle a\rangle=$ NumberField $(f)$

return K.class_number ()

Next, we define the function test (F). It returns True if and only if there is an $\epsilon$ positive generator of the different of $K_{0}$ for some $\epsilon$ with a single -1 . In paragraph one of the code, a generator d0 of the different and generators $u[0], \ldots, u[3]$ for the free part of the group of units are found for $K_{0}$.

In the second paragraph, a search is conducted over the set $\{0,1\}^{5}$ in $i, j, k, \ell, m$ space. For each element of the set, we ask whether $d=(-1)^{i} d_{0} u_{0}^{j} u_{1}^{k} u_{2}^{\ell} u_{3}^{m}$ is $\epsilon$ positive. If a lattice point passes the test, the function test returns True. If no lattice point passes the test, it returns False. The validity of the test rests on the 
fact that the positivity properties of $\tau_{r}(d)$ depend only on the exponents $j, k, \ell, m$ modulo 2 .

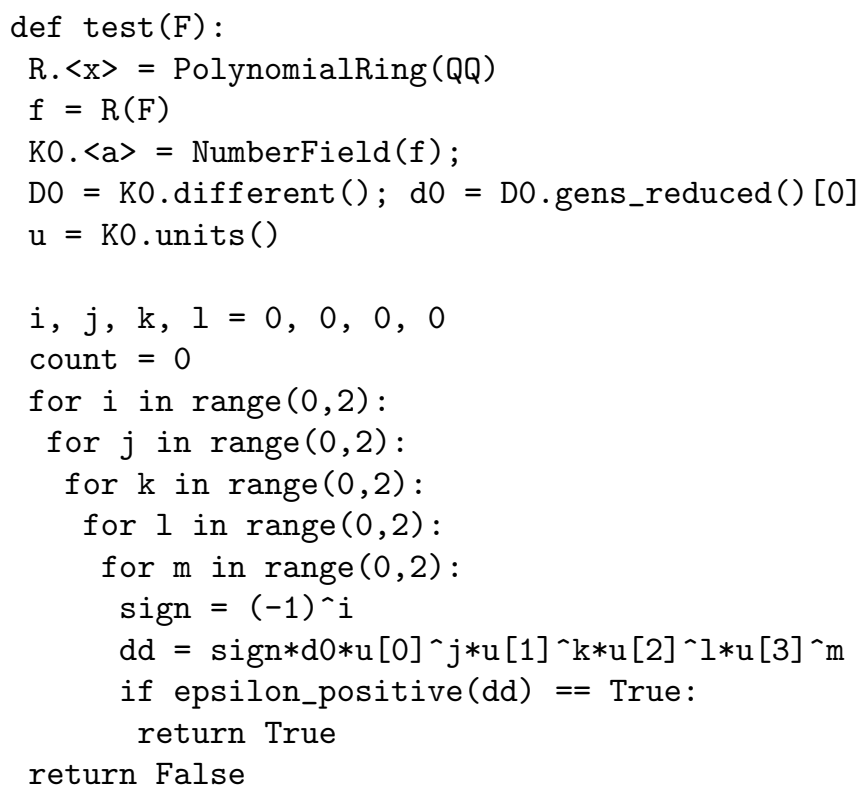

The test for $\epsilon$-positivity is carried out by the function below:

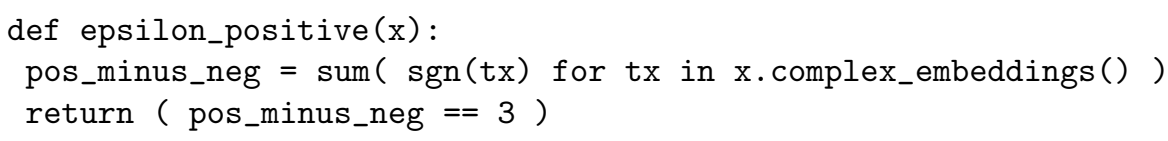

Finally, we enumerate the totally real quintic fields of discriminant less than $N$, applying the above test to each and collecting various statistics.

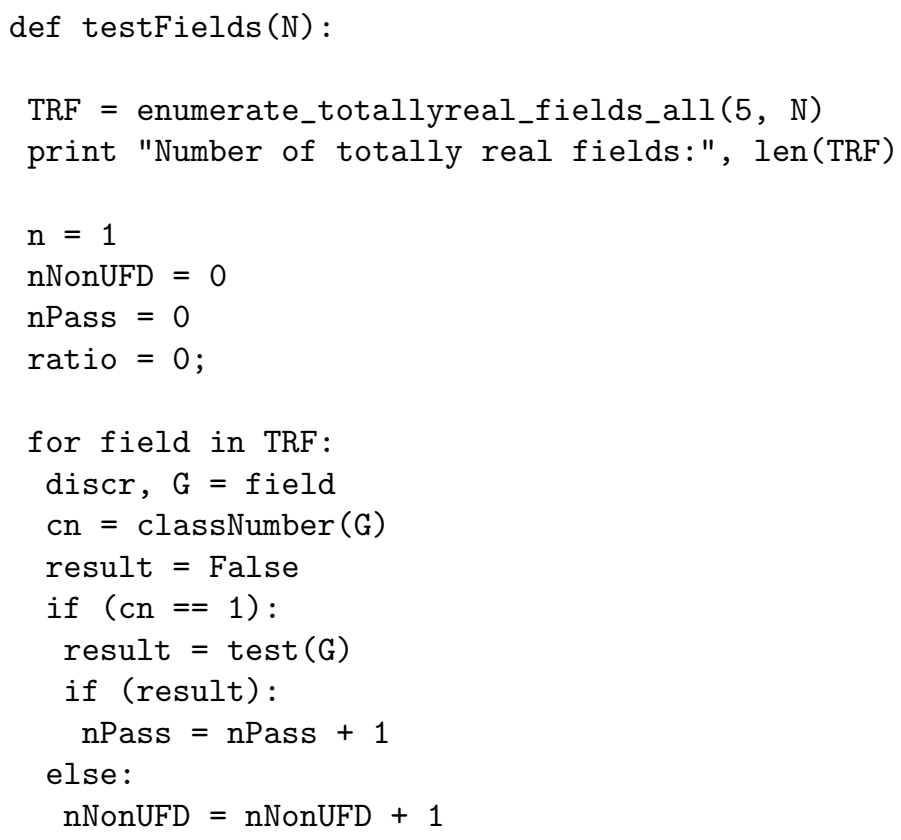




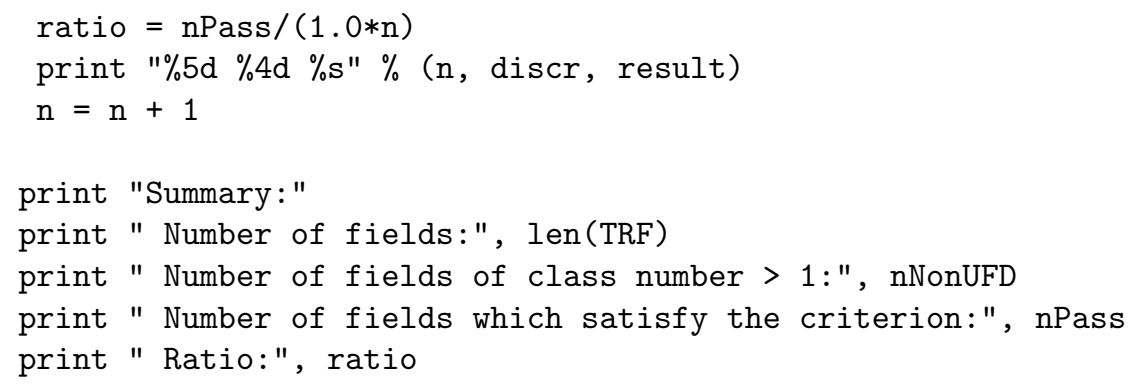

To run the test on fields of discriminant $<10^{6}$, run the command testFields (10^6).

\section{Problems}

As promised in the introduction, we list some problems suggested by the analogy between the period map $\mathcal{P}$ for cubic surfaces and the classical period map for cubic curves.

- Find explicit values of the period map. This can be interpreted in two ways: the actual period vector $\mathcal{P}(F, \gamma) \in \mathbb{C}^{1,4}$ as in (2.7), or as a period ratio, namely the equivalence class of $\mathcal{P}(F, \gamma)$ in $\mathbb{B}^{4}$ or in $\Gamma \backslash \mathbb{B}^{4}$. We remark that nothing seems to be known about the periods $\mathcal{P}(F, \gamma)$. The known period ratios from $\S 11$ of [3, (2.6) and (2.7) above are found by symmetry considerations. The Fermat and Clebsch surfaces are uniquely determined by their automorphism group and this group is generated by reflections. This suggests an easier subproblem:

- Find values of the period map for all surfaces with symmetries. These surfaces are totally classified. One special family of such surfaces that should be more tractable is the following:

- Find the values of the period map for all cyclic cubic surfaces. Express them as explicit functions of the periods of the corresponding cubic curves. A cyclic cubic surface means a surface $S \subset \mathbb{P}^{3}$ given by an equation $x_{3}^{3}=f\left(x_{0}, x_{1}, x_{2}\right)$, where $f\left(x_{0}, x_{1}, x_{2}\right)$ is a nonsingular cubic form; in other words, $S$ is the cyclic cover of $\mathbb{P}^{2}$ branched along the cubic curve $f=0$.

- Study special values of the period map. We mean the situation when the abelian variety has complex multiplication.

- Find an explicit inverse to the period map. We mean: given $b \in \mathbb{B}^{4}-\mathcal{H}$, find an explicit cubic form $F$ so that the class of $\mathcal{P}(F, \gamma)$ is $b$. In principle this can be done using suitable theta functions. Allcock and Freitag [4] and Matsumoto and Terasoma [7] have given projective embeddings of $\Gamma \backslash \mathbb{B}^{4}$ with image an algebraic embedding of (a covering of) $\mathcal{M}_{s t}$. In this way one obtains formulas for algebraic invariants of $F$ in terms of $b$, but we are not aware of explicit formulas for a cubic form.

- Study the algebraic and arithmetic nature of the period map. Both the domain and target of $\mathcal{P}: \mathcal{M}_{s t} \longrightarrow \Gamma \backslash \mathbb{B}^{4}$ are algebraic varieties and $\mathcal{P}$, in spite of its transcendental definition, is an algebraic map. See [1] for more information and for progress on this question. 


\section{ACKNOWLEDGEMENTS}

The authors are indebted to Madhav Nori, who explained to them how to use the different ideals to check whether the abelian variety defined by a number field is principally polarized. They would also like to express their thanks to the referee, whose comments greatly improved this paper.

\section{REFERENCES}

1. J. Achter, Arithmetic Torelli maps for cubic surfaces and threefolds, arXiv:1005.2131v3 [math.AG], 29 Feb. 2012.

2. J. Achter, On the abelian fivefolds attached to cubic surfaces, arXiv:1208.2974v1 [mathAG], 14 Aug. 2012.

3. D. Allcock, J. Carlson, and D. Toledo, The complex hyperbolic geometry of the moduli space of cubic surfaces, J. Algebraic Geom. 11 (2002), 659-724. MR1910264 (2003m:32011)

4. D. Allcock and E. Freitag, Cubic surfaces and Borcherds products, Comment. Math. Helv. 77 (2002), 270-296. MR:1915042(2004c:14067)

5. A. Beauville, Moduli of cubic surfaces and Hodge theory (after Allcock, Carlson, Toledo), Géométries à courbure négative ou nulle, Sémin. Congr. 18, Soc. Mat. France, Paris, 2009, 445-466. MR2655320(2011g:32022)

6. S. Lang, Complex Multiplication (Grundlehren der mathematischen Wissenschaften 255), Springer-Verlag, 1983, 185 pp. MR713612 (85f:11042)

7. K. Matsumoto and T. Terasoma, Theta constants associated to cubic threefolds. J. Algebraic Geom. 12 (2003), 741-775. MR.1993763 (2004h:14041)

8. D. Mumford, Abelian Varieties, Oxford University Press, 1970, 279 pp. MR0282985 (44:219)

9. D. Mumford, A note on Shimura's paper "Discontinuous Groups and Abelian Varieties", Math. Ann. 181 (1969), 345-351. MR0248146 (40:1400)

10. Jürgen Neukirch, Algebraic Number Theory, Springer-Verlag (Grundlehren der mathematischen Wissenschaften 322), 1999, 571 pp. MR1697859 (2000m:11104)

11. M. Waldschmitt, Transcendence of Periods: The State of the Art, Pure and Applied Mathematics Quarterly, Volume 2, Number 2 (Special Issue: In honor of John H. Coates, Part 2 of 2), 435-463, 2006. MR2251476(2007d:11083)

Clay Mathematics Institute, One Bow Street, Cambridge, Massachusetts 02138

E-mail address: jcarlson@claymath.org

Current address: 25 Murray Street, Apt. 7G, New York, New York 10007

E-mail address: jxxcarlson@mac.com

Department of Mathematics, University of Utah, Salt Lake City, Utah 84112

E-mail address: toledo@math.utah.edu 